\title{
Risk Reducing Surgery in Carriers with Double Het- erozygosity for BRCA1 and BRCA2 Mutations
}

Woo Sung Hong ${ }^{1}$ Ku Sang Kim", Yong Sik Jung', Seok Yun Kang², Doo Kyoung Kang ${ }^{3}$, Tae Hee Kim³ ${ }^{3}$ Hyunee Yim ${ }^{4}$, Mison Chun ${ }^{5}$, Myong Chul Park ${ }^{6}$ and Suk-Joon Chang

Departments of ${ }^{1}$ Surgery, ${ }^{2}$ Hematology - Oncology, ${ }^{3}$ Radiology, ${ }^{4}$ Pathology, ${ }^{5}$ Radiation Oncology, ${ }^{6}$ Plastic and Reconstructive Surgery, and ${ }^{7} \mathrm{Ob}-$ stetrics and Gynecology, Ajou University School of Medicine, Suwon, Korea

Among the treatment options for $B R C A$ mutation carriers, risk reducing surgery is the most effective. However, this procedure has been rarely performed in Korea. Interestingly, our case showed double heterozygosity for BRCA1 and BRCA2 mutations. The patient was diagnosed with left renal cancer and left breast cancer at 45-years-of-age, 4 years before risk reducing surgery. The patient received left radical nephrectomy and left partial mastectomy with axillary lymph node dissection. After pretest counseling, the patient underwent genetic testing that identified BRCA1 and BRCA2 mutations. After post-test counseling, the patient decided on intensive surveillance. At 49-years-of-age, the patient was newly diagnosed with contralateral breast cancer. Treatment options were discussed once again. We performed bilateral total mastectomy with immediate reconstruction and prophylactic bilateral salpingo-oophorectomy after multidisciplinary discussion. The patient has been satisfied with the results of surgery. We think this procedure is a recommendable treatment option for BRCA mutation carriers.

Key Words: Breast neoplasms, BRCA1 gene, BRCA2 gene, Mastectomy

\section{Introduction}

Breast cancer is the most common female carcinoma in the world. Hereditary breast cancer comprises 5-10\% of the cases of breast cancer, and are mainly caused by germline mutations of the $B R C A$ gene. ${ }^{1-3)}$ Since the introduction of DNA testing for detection of BRCA1/2 gene mutations, many researchers have attempted to explain the clinical features, prognosis, and risk reducing strategies of $B R C A$ mutation carriers. The lifetime risk of breast cancer has been estimated to be up to $84 \%$, and the risk of ovarian cancer ranges from $36-63 \%$ for BRCA1 and 10-27\% for BRCA2 mutation carriers. ${ }^{1-3)}$ The characteristics of hereditary breast cancer are young age onset, frequently bilateral occurrence, high risk of ovarian and other organ cancers, and family historyamong multiplegenerations. The prognosis of $B R C A$ associated breast cancer is uncertain. It has been suggested that the prognosis is similar to that of sporadic breast cancer, while others have opined that the prognosis of $B R C A$ associated breast cancer is worse than sporadic cancer. ${ }^{2,3)}$ Treatment options for $B R C A$ mutation carriers are intensive surveillance, chemoprevention, and risk reducing surgery. Although none of those treatments completely protectsagainst breastcancer, risk reducing surgery is known to be the most effective strategy. ${ }^{1,2}$ However, for many reasons, fewer women in Asian countries choose risk reducing surgery compared to their counterparts in Western countries. Recently, we experienced a case of risk reducing surgery in a carrier with double heterozygosity for $B R C A 1$ and BRCA2 mutations. We herein report this case.

Received: 10 March 2012, Revised: 12 May 2012, Accepted: 16 May 2012, Published: 30 June 2012

*Corresponding author: Ku Sang Kim

Department of Surgery, Ajou University School of Medicine, San 5, Woncheon-dong, Yeongtong-gu, Suwon, 443-721, Korea

Tel: +82-31-219-5200, Fax: +82-31-219-5755, E-mail: ideakims@gmail.com

(c) This is an open-access article distributed under the terms of the Creative Commons Attribution Non-Commercial License (http://creativecommons.org/licenses/by-nc/3.0/) which permits unrestricted non-commercial use, distribution, and reproduction in any medium, provided the original work is properly cited.

(c) Copyright 2012 by the Korean Society of Medical Genetics 


\section{Case Report}

\section{Past history and pre-test genetic counseling}

The patient was referred to our hospital for a left renal mass that had been incidentally found 4 years before the risk reducing surgery, at 45-years-of-age during a regular medical examination. Under the diagnosis of renal cell carcinoma, the patient underwent several radiologic examinations for cancer workup, and positron emission tomography/computed tomography (PET/CT) of the torso revealed focally increased fluorodeoxyglucose (FDG) uptake in the left breast and left axillary area. To rule out double primary cancer, a breast examination was performed using breast ultrasonography and mammography. A $0.7 \mathrm{~cm}$ sized left breast mass and $3.7 \mathrm{~cm}$ sized ipsilateral axillary lymph node were found, and invasive ductal carcinoma was confirmed by 14-gauge core needle biopsy. On August 14, 2007, wide local excision of the left breast with axillary lymph node dissection was performed by breast surgeons, and left radical nephrectomy was performed by urologists. The result of the breast histopathology was invasive ductal carcinoma, T1N1M0 (0.7 cm tumor, two metastatic lymph nodes identified among 15 dissected nodes), which was negative for estrogen receptor, positive for progesterone receptor, and negative for C-erbB2. The result of the kidney histopathology was renal cell carcinoma, clear cell type, Fuhman's nuclear grade 3, confined to the capsule and free of tumor metastasis to regional lymph nodes. The patient received adjuvant chemotherapy (AC-T regimen: four cycles of doxorubicin $50 \mathrm{mg} / \mathrm{m}^{2}$ and cyclophosphamide $500 \mathrm{mg} / \mathrm{m}^{2}$, followed by four cycles of paclitaxel $175 \mathrm{mg} / \mathrm{m}^{2}$ at an interval of 3 weeks), adjuvant radiotherapy (total 5900 cGyat leftbreastand boost), and adjuvanthormone therapy (toremifene 40 mg once a day since July 23,2008 ). The patient was scheduled for routine follow-up. To the patient's knowledge, no family member had been diagnosed with any cancer (Fig. 1). However, since the patient had a personal history of multiple primary cancers, we informed her about hereditary breast cancer. The patient underwent genetic counseling, which included information regarding the risks, limitations, and possible events of $B R C A$ mutation. The patient decided to receive genetic testing, which was performed after informed consent was provided.

\section{Genetic testing and post-test genetic counseling}

Genetic testing using the method of direct sequencing was performed in September 2008. Both BRCA1 and BRCA2 gene mutations were identified in this test. As the 509th base of exon 7 on the BRCA1 gene, cytosine, was substituted with adenine [BRCA1 $509 C>A$ (c.390C $>A)]$, and the 130th amino acid, tyrosine, was transformed to a stop codon (p.Tyr130X). In addition, the 3246th base of exon 11 on the BRCA2 gene, adenine, was deleted [BRCA2 3246delA (c.3018delA)], which produced a frame shift change, and the 1042th amino acid was transformed to a stop codon

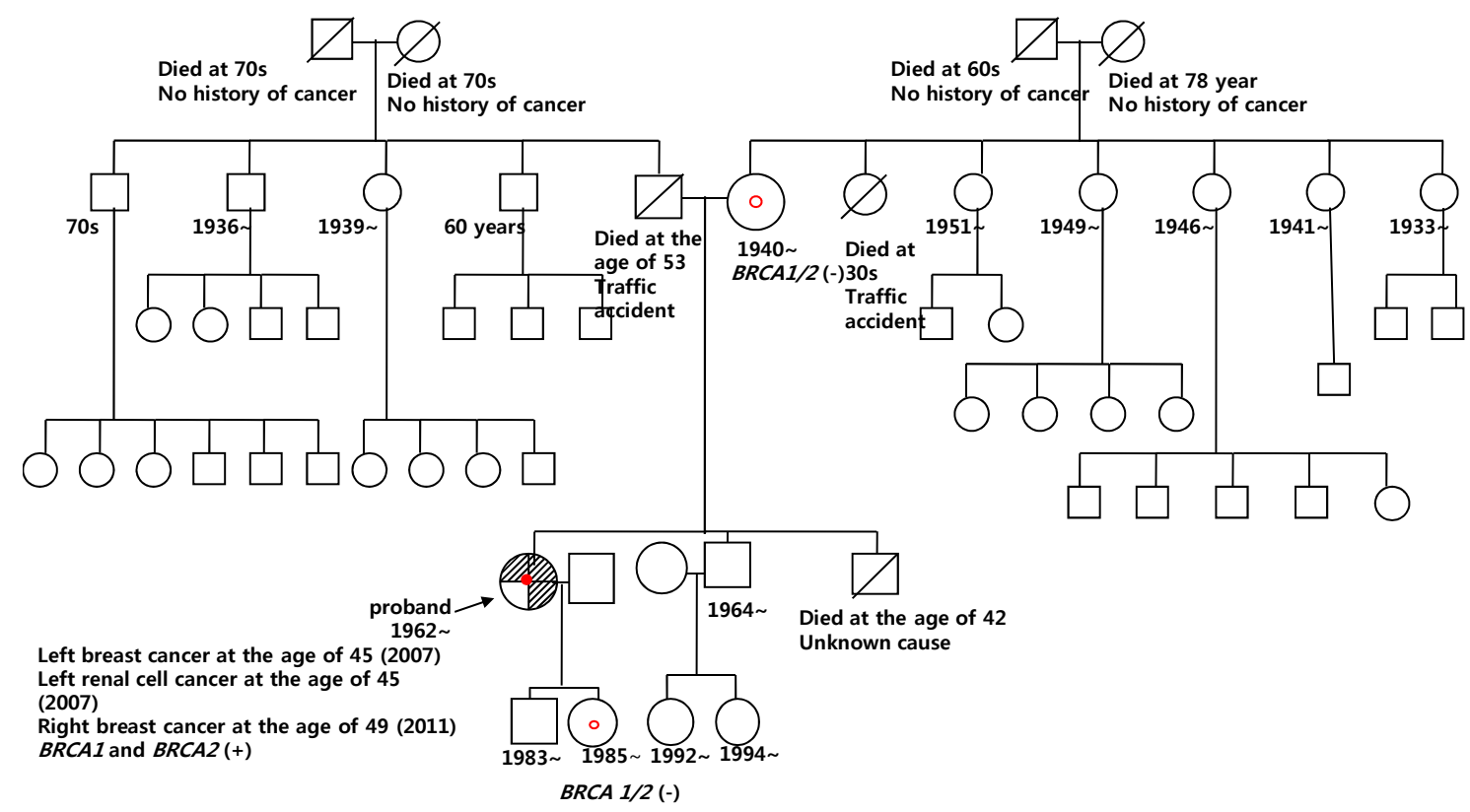

Fig. 1. Pedigree of the patient. There was no family history. The arrow indicates the patient. BRCA1 and BRCA2 mutations were identified upon examination of the patient. Site specific genetic testing performed on her mother and daughter was negative. 
(p.G1007VfsX36) (Fig. 2). All sequence variants were named according to the nomenclature used by both Human Genome Variation Society (HGVS) and Breast Cancer Information Core (BIC) database. After the BRCA mutation was identified, site-specific genetic testing was performed on the patient's mother and daughter with the agreement of family members. The results were both negative. We recommended evaluating the patient's paternal family members to ascertain the origin of mutation, but the family members did not consent. We explained the risk of local recurrence and occurrence of contralateral breast cancer and ovarian cancer, and educated the patient concerning the benefits and drawbacks of each treatment option. The patient had been receiving hormonal therapy and chose intensive surveillance. The patient had also received routine examinations for 4 years, with mammography and breast ultrasonography being performed at 6-month intervals. As well, FDG-PET/CT (or bone scan with abdominal ultrasound) had been performed at 6-month intervals. On June 08, 2011, a newly developed $0.7 \mathrm{~cm}$

\section{Control (-)}

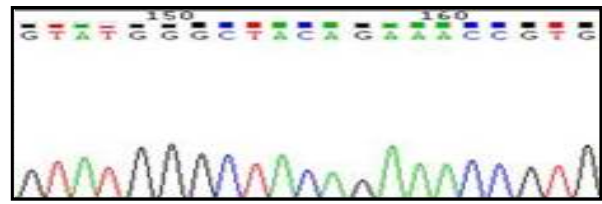

JB2189
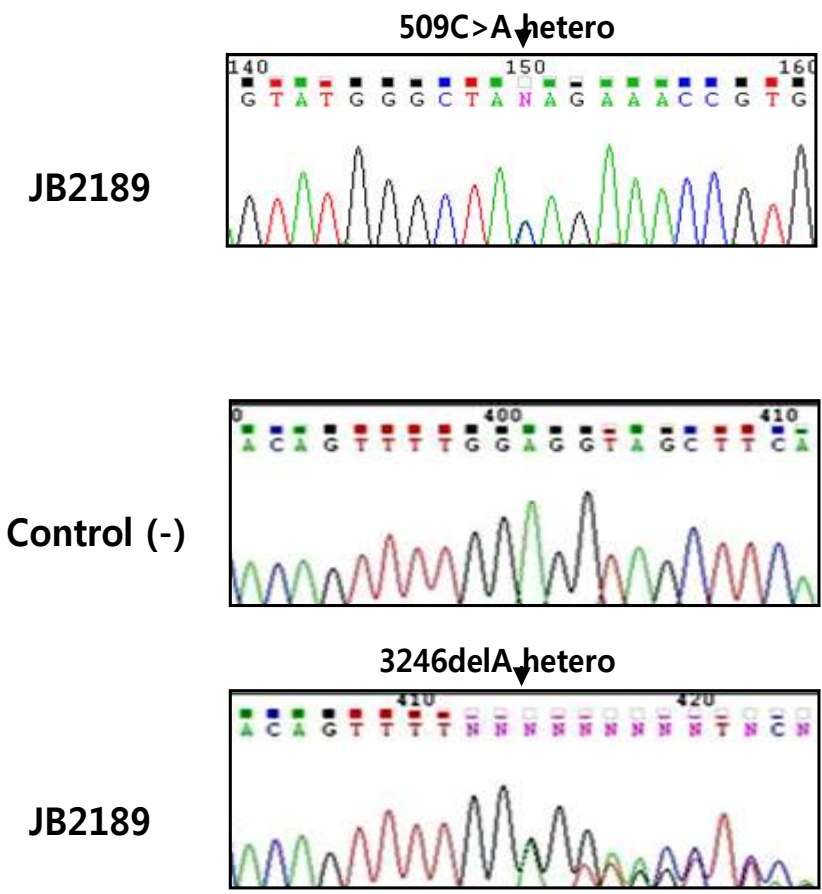

Fig. 2. DNA sequencing data. Double heterozygosity of $B R C A 1$ and $B R C A 2$ was identified. The 509th base of exon 7 on the BRCA1 gene was substituted, and the 3246th base of exon 11 on the BRCA2 gene was deleted [BRCA1 509C $>\mathrm{A}(\mathrm{c} .390 \mathrm{C}>\mathrm{A})$ and BRCA2 3246delA (c.3018delA)]. sized right breast mass was identified by breast ultrasonography. Invasive ductal carcinoma was confirmed by 14-gauge core needle biopsy. We recommended risk reducing surgery once again. The patient consented to a bilateral total mastectomy with immediate reconstruction and prophylactic bilateral salpingooophorectomy.

\section{Pre-operative psychological evaluation}

We asked questions in the psychological evaluation and requested a body-image self-estimate, as reported by Kim et al. ${ }^{4)}$ The Beck Depression Inventory (BDI) was 13 points, which was indicative of mild depression status. The score of a re-evaluation of life orientation test (LOT-R) was 17 points out of 24. Although there was not a defined cut-off value of LOT- $R$, the patient scored above the median value, which meant that the patient was relatively optimistic. Among the evaluation contents of the StateTrait Anxiety Inventory (STAI), the trait anxiety score and stat anxiety score were 35 and 52 points, respectively (range, 0-60). Each score meant 'not anxious'. Among a 36-item short form survey instrument (SF-36) as an index for evaluation of quality of life, the physical component score and mental component score were 83.8 and 65.6, respectively. The results of the SF-36 were more than the median value (50 points). The Sociocultural Attitudes Towards Appearance Questionnaire (SATAQ-3) and Multidimensional Body-Self Relations Questionnaire (MBSRO) as indices to evaluate attitudes regarding appearance and body image were performed. The total score of the SATAQ- 3 was 83 points out of 150 (Internalization-General, 29 points out of 45; Internalization-Athlete, 16 points out of 25; Pressures Items, 23 points out of 35; Information Items, 31 points out of 45). The results meant that socio-cultural influences on appearance were lower than the normal standard value. The patient had lower internalization of social and cultural standard attitudes regarding appearance than the general population. In other words, the patient was seldom influenced by surroundings, fashions, mass media, and others around her. The score of MBSRQ was 191 points out of 345 (mean value 2.77). It was lower than the general population or other patients with breast cancer. According to this test, the patient generally had a tendency to set a lower value on appearance, fitness, health, and body image.

\section{Surgical plan and written consent form}

Each clinical department, including the Department of Breast Surgery, Department of Obstetrics and Gynecology, and Depart- 
ment of Plastic and Reconstructive Surgery, participated in a multidisciplinary discussion on the surgical plan. The advantage and disadvantage of each surgical option were explained. The patient decided on bilateral total mastectomy with immediate reconstruction and prophylactic bilateral salpingo-oophorectomy. The surgical methods, complications, and benefits were explained several times. The patient and family members agreed with the plan and signed the consent form.

\section{Operative finding and post-operative course}

Risk reducing surgery was conducted on July 12, 2011. Under general anesthesia, a nipple areola complex sparing subcutaneous mastectomy of both breasts and right sentinel lymph node biopsy were performed. The total removed volume of the rightand left breast tissue was $300 \mathrm{gm}$ and $275 \mathrm{gm}$, respectively. After mastectomy, immediate reconstruction was performed with a cohesive gel-filled implant (325 mL, moderate profile; Mentor) by the plastic surgery team, and then laparoscopic guided prophylactic bilateral salpingooophorectomy was performed by the obstetrics and gynecology team. The histopathology results reported the presence of a $1.0 \mathrm{~cm}$ invasive ductal carcinoma at the right breast with negative ipsilateral sentinel lymph node. Immunohistochemistry examinations were negative for estrogen receptor, progesterone receptor, and C-erbB2. No abnormal pathologic findings were found in the left breast and both ovaries and salpinges. The patient was relatively satisfied with the result of surgery at an outpatient interview. The patient received adjuvant chemotherapy (CMF regimen: six cycles of cyclophosphamide $600 \mathrm{mg} / \mathrm{m}^{2}$, methotrexate $40 \mathrm{mg} / \mathrm{m}^{2}$, and fluorouracil $600 \mathrm{mg} / \mathrm{m}^{2}$ at 3 -weekinterval).

\section{Discussion}

The case in this report showed double heterozygosity for BRCA1 and BRCA2 mutations, which is an extremely rare phenomenon. Almost all cases have been in the Ashkenazi Jewish population. ${ }^{5)}$ The clinical and phenotypic characteristics of double heterozygosity for BRCA1 and BRCA2 mutations are not completely understood, although a few cases have been reported in the literature. In 2005, Leegte et al. ${ }^{5}$ presented four double heterozygosity cases and reviewed all published data. Approximately 34 cases with double heterozygosity from 25 different families were included in this report. The incidence was 1.8\% among Ashkenazi Jewish mutation carriers. The phenotypic expression varied from early onset breast and ovarian cancer to cancer-free survival at age 70 , which was similar to a general $B R C A$ mutation carrier. In this report, the mean age at diagnosis for breast cancer and ovarian cancer was 41.1 and 45 years, respectively, and the cumulative life time risk of cancer was approximately $80 \%$. The analysis of loss of heterozygosity in both breast and ovarian tumors did not demonstrate a predominant contribution of either BRCA1 or BRCA2 gene oncogenesis. The family history, which was available in only 18 out of the 25 family members, was observed in 13 members with a maternal history, one with paternal history, and four with both sides. The authors emphasized that the analysis of all founder mutations should be performed when a BRCA1 or BRCA2 mutation is detected in an index case and suggested that a woman with double heterozygosity could be offered the usual risk reducing strategy.

Recently, five Korean cases of double heterozygosity for BRCA1 and BRCA2 mutations were reported. ${ }^{6}$ In Korea, there has been no report of Ashkenazi founder mutations. The prevalence of double heterozygosity among Korean BRCA mutation carriers is about $1.2 \%$. The phenotypic expression was similar to that of BRCA1 mutation carrier. The mean age at diagnosis of breast cancer was 33 years, and all patients who had a family history acquired it from the maternal side. Data from an Italian report was similar to Korean result. ${ }^{7)}$ The incidence of double heterozygosity was $0.62 \%$ of mutated individuals. Mean ages of patients at the time of breast and ovarian cancer diagnosis were 42.7 and 48.6, respectively. The breast cancers were all high grade, negative for hormone receptors and HER2 expression, and had a high proliferative index. Although no patient developed contralateral breast cancer, $75 \%$ had both a breast and an ovarian cancer. The occurrence of gastrointestinal tumors was frequently observed in the family members. Although most studies had small sample sizes and lacked a long-term follow up, risk reducing surgery should be considered in non-Ashkenazi cases because they present more severe clinical features.

The patient in this report had received breast conserving surgery and radiation therapy for left breast cancer before $B R C A$ mutation was confirmed. It was possible that the newly developed cancer could also be treated by breast conserving surgery, if the patient was not a $B R C A$ mutation carrier. But, local recurrence after breast conserving surgery in $B R C A$ mutation carriers occurs more frequently. ${ }^{8-10)}$ Garcia-Etienne et al. compared BRCA mutation carriers receiving breast conserving surgery and standard radiotherapy with patients of sporadic breast cancer receiving the same treatment. ${ }^{9)}$ The 10-year cumulative incidence of ipsilateral breast tumor recurrence was 27\% for mutation carriers and 4\% for sporadic controls, and the 10-year cumulative incidence of 
contralateral breast cancer was 25\% for mutation carriers and $1 \%$ for sporadic controls. Whether carriers who had undergone oophorectomy were removed or not from analysis, this trend was notmodified.

Sentinel lymph node biopsy is an acceptable and relatively safe procedure, making it possible to avoid unnecessary axillary dissection. However, this procedure in the setting of prophylactic mastectomy remains unclear. Occult breast cancer was identified in a prophylactic mastectomy specimen, and crossover metastasis to the opposite axilla could have occurred without contralateral breast cancer." 0 n the other hand, although a sentinel lymph node biopsy has a lower risk of complications than the routine procedure of axillary dissection, it is not free from lymphedema, uncomfortable pain, sensory loss, or limited range of motion. A recent study reported that sentinel lymph node biopsy of the unaffected breast was beneficial in only $2.8 \%$ at the time for prophylactic mastectomy, and almost cases of a positive sentinel lymph node were locally advanced cancer. ${ }^{11)}$ For this reason, some have recommended that sentinel node biopsy of the unaffected breast should be performed only in locally advanced cases or with lesions detected preoperatively on magnetic resonance imaging (MRI). ${ }^{11)}$ We performed only right sentinel lymph node biopsy (affected side), because we evaluated the absence of occult cancer on preoperative $\mathrm{MRI}$, and the axillary node of the opposite site was completely dissected during the previous operation.

Prophylactic bilateral salpingo-oophorectomy is commonly performed laparoscopically. The risk of ovarian cancer as well as breast cancer is reduced by prophylactic bilateral salpingooophorectomy. Although primary peritoneal carcinomatosis after surgery has been reported, it is an effective and potential prophylactic strategy for BRCA mutation carriers. Recently published data demonstrated that prophylactic salpingo-oophorectomy can significantly decrease the rates of breast and ovarian cancer occurrence, breast and ovarian cancer specific mortality, and all cause death. ${ }^{12)}$ However, some patients suffered from surgeryinduced early menopausal symptoms. Although hormone replacement therapy for BRCA mutation carriers who received prophylactic salpingo-oophorectomy is controversial, short-term hormone replacement therapy after prophylactic salpingo-oophorectomy has proved not to be negative for preventive effect and should be carefully considered. ${ }^{13)}$

Nevertheless, risk reducing surgery has been rarely performed in Korea, even though practice patterns for managing hereditary breast cancer were changed by many Korean physicians after the Korean Hereditary Breast Cancer Study. ${ }^{14)}$ Only two cases have been reported in the literature. ${ }^{4,15}$ Most Korean BRCA mutation carriers have preferred intensive surveillance. Factors that contributed to the decision concerning prophylactic mastectomy were not associated with an increased recognition of the breast cancer risk by the patient, but were instead associated with ethnicity, age, prior cancer history, having young children, and greater awareness of the genetic natureand other treatmentfactors, such as immediate reconstruction, preoperative $\mathrm{MRI}$, and unsuccessful attempts at breast conservation. ${ }^{16)}$ A prospective study that compared patient satisfaction before and 1 year after surgery reported that there were no negative effects on anxiety, depression, and quality of life, but negative effects were found in sexuality and body image. ${ }^{17}$ The most beneficial factor contributing to patient satisfaction is a reduction in breast cancer related concerns, and the most common reason for regret after prophylactic mastectomy is a poor cosmetic outcome. ${ }^{18,19)}$ In our case, the patient was evaluated concerning psychological factors, quality of life, and body image before the surgery. Broadly positive results were observed in these evaluations, and the patient was relatively satisfied with the results of surgery at an outpatient department interview. Although it is necessary to follow-up on the patient's satisfaction and quality of life, it is thought that risk reducing surgery is an acceptable procedure in $B R C A$ mutation carriers, especially carriers in double heterozygosity of $B R C A 1$ and $B R C A 2$ mutation, because of aggressive phenotypic feature in non-Ashkenazi Jewish population.

\section{Acknowledgement}

This case report was supported by National R\&D Program for Cancer Control of the Ministry of Health and Welfare (Grant number 1020350).

\section{References}

1. Rebbeck TR, Friebel T, Lynch HT, Neuhausen SL, van't Veer L, Garber JE, et al. Bilateral prophylactic mastectomy reduces breast cancer risk in BRCA1 and BRCA2 mutation carriers: the PROSE Study Group. J Clin Oncol 2004;22:1055-62.

2. van Roosmalen MS, Verhoef LC, Stalmeier PF, Hoogerbrugge $N$, van Daal WA. Decision analysis of prophylactic surgery or screening for BRCA1 mutation carriers: a more prominent role for oophorectomy. J Clin Oncol 2002;20:2092-100.

3. Grann VR, Jacobson JS, Whang W, Hershman D, Heitjan DF, Antman $\mathrm{KH}$, et al. Prevention with tamoxifen or other hormones versus prophylactic surgery in BRCA1/2-positive women: a decision analysis. Cancer 
J Sci Am 2000;6:13-20.

4. Kim KS, Kim S, Han SA, Kang E, Jeon YT, Ha TH, et al. Contralateral prophylactic mastectomy and prophylactic salphingo-oophorectomy in a BRCA1-positive breast cancer patient: A case report. J Breast Cancer 2008;11:218-22.

5. Leegte $B$, van der Hout AH, Deffenbaugh AM, Bakker MK, Mulder IM, ten Berge $A$, et al. Phenotypic expression of double heterozygosity for BRCA1 and BRCA2 germline mutations. J Med Genet 2005;42:e20.

6. Noh JM, Choi DH, Nam SJ, Lee JE, Kim JW, Kim SW, et al. Characteristics of double heterozygosity for BRCA1 and BRCA2 germline mutations in Korean breast cancer patients. Breast Cancer Res Treat 2012;131:217-22.

7. Zuradelli $M$, Peissel $B$, Manoukian S, Zaffaroni $D$, Barile $M$, Pensotti V, et al. Four new cases of double heterozygosity for BRCA1 and BRCA2 gene mutations: clinical, pathological, and family characteristics. Breast Cancer Res Treat 2010;124:251-8.

8. Haffty BG, Harrold E, Khan AJ, Pathare P, Smith TE, Turner BC, et al. Outcome of conservatively managed early-onset breast cancer by BRCA1/2 status. Lancet 2002;359:1471-7.

9. Garcia-Etienne CA, Barile M, Gentilini OD, Botteri E, Rotmensz N, Sagona A, et al. Breast-conserving surgery in BRCA1/2 mutation carriers: are we approaching an answer? Ann Surg Oncol 2009;16:3380-7.

10. Seynaeve $C$, Verhoog LC, van de Bosch LM, van Geel AN, Menke-Pluymers $M$, Meijers-Heijboer EJ, et al. Ipsilateral breast tumour recurrence in hereditary breast cancer following breast-conserving therapy. Eur J Cancer 2004;40:1150-8
11. Zhou WB, Liu XA, Dai JC, Wang S. Meta-analysis of sentinel lymph node biopsy at the time of prophylactic mastectomy of the breast. Can J Surg 2011:54:6010-6.

12. Domchek SM, Friebel TM, Singer CF, Evans DG, Lynch HT, Isaacs $C$, et al. Association of risk-reducing surgery in BRCA1 or BRCA2 mutation carriers with cancer risk and mortality. JAMA 2010;304:967-75.

13. Eisen A, Lubinski J, Gronwald J, Moller P, Lynch HT, Klijn J, et al. Hormone therapy and the risk of breast cancer in BRCA1 mutation carriers. J Natl Cancer Inst 2008; 100:1361-7.

14. Kang E, Ahn SH, Noh WC, Noh DY, Jung Y, Kim LS, et al. The change of practice patterns of the hereditary breast cancer management in Korea after the Korean Hereditary Breast Cancer Study. J Breast Cancer 2010;13:418-30.

15 Choi MY, Lee JE, Kim SW, Lee SK, Hur SM, Kim S, et al. Incidental detection of ductal carcinoma in situ of the breast from bilateral prophylactic mastectomy of asymptomatic BRCA2 mutation carrier: A case report. J Breast Cancer 2010;13:311-7.

16. King TA, Sakr R, Patil S, Gurevich I, Stempel M, Sampson M, et al. Clinical management factors contribute to the decision for contralateral prophylactic mastectomy. J Clin Oncol 2011;29:2158-64.

17. Brandberg $Y$, Sandelin $K$, Erikson $S$, Jurell $G$, Liljegren $A$, Lindblom $A$, et al. Psychological reactions, quality of life, and body image after bilateral prophylactic mastectomy in women at high risk for breast cancer: a prospective 1-year follow-up study. J Clin Oncol 2008;26: 3943-9. 